\title{
Intrinsic, deductive, explicit, and algorithmic characterization of the Szekeres-Szafron solutions
}

\author{
Joan Josep Ferrande* \\ Departament d'Astronomia i Astrofísica, Universitat de València, E-46100 Burjassot, València, Spain. \\ Juan Antonio Sáez \\ Departament de Matemàtiques per a l'Economia i l'Empresa, \\ Universitat de València, E-46071 València, Spain.
}

(Dated: July 27, 2021)

\begin{abstract}
We write the known invariant definition of the Szekeres-Szafron family of solutions in an intrinsic, deductive, explicit and algorithmic form. We also intrinsically characterize the two commonly considered subfamilies, and analyze other subclasses, also defined by first-order differential conditions. Furthermore, we present a Rainich-like approach to these metrics.
\end{abstract}

\section{INTRODUCTION}

The Szekeres-Szafron family of solutions is known as one of the most significant inhomogeneous cosmological models [1 3]. These metrics were obtained by Szafron [4] as the generalization for a nonvanishing pressure of the Szekeres dust solutions [5]. The symmetries and other physical and geometric properties of the Szekeres dust models have been widely studied [1, 2, 6 6 9] (see also the recent papers [10, 11] and references therein).

On the other hand, Szafron [4] also extended the invariant characterization by Wainwright [12] of the Szekeres solutions to the nonvanishing pressure models:

Invariant characterization 1 [4]: A Szekeres-Szafron metric is characterized by the following conditions: (i) it is a perfect fluid solution with a geodesic and irrotational unit velocity; (ii) the Weyl tensor is Petrov-Bel type D and the velocity of the fluid lies in the two-plane $\Pi$ spanned by the two null principal directions; (iii) any vector in the two-plane $\Pi^{\perp}$ is an eigenvector of the shear; (iv) the two-plane $\Pi$ admits orthogonal two-surfaces.

Note that the above statement (i) is a first-order differential condition for the Ricci tensor. The second one is algebraic for both the Ricci and the Weyl tensors. The third one is algebraic for the Weyl tensor and a first-order differential condition for the Ricci tensor. And (iv) is a first-order differential condition for the Weyl tensor. So, characterization 1 imposes first-order differential conditions on the Ricci and Weyl tensors.

In a later paper Szafron and Collins [13] offered an alternative invariant characterization following from their study of the inhomogeneous cosmological models with intrinsic symmetries [14].

Invariant characterization 2 [13]: A Szekeres-Szafron metric is characterized by the following conditions: (i) it is a perfect fluid solution with a geodesic and hypersurface orthogonal unit velocity; (ii) each space-like hyper-

\footnotetext{
* Also at Observatori Astronòmic, Universitat de València, E-46980 Paterna, València, Spain; joan.ferrando@uv.es.
}

surface orthogonal to the unit velocity has an induced conformally flat metric $\gamma$; (iii) the Ricci tensor of $\gamma$ has a double eigenvalue; (iv) the shear tensor of the fluid has a double eigenvalue.

Now, statements (i) and (iv) are first-order differential conditions for the Ricci tensor of the spacetime metric. Moreover, as the metric $\gamma$ is algebraic in the Ricci tensor, (ii) and (iii) are differential constraints for the Ricci tensor, of third and second order, respectively. Consequently, characterization 2 imposes third order differential conditions solely in terms of the Ricci tensor.

Finally, a third invariant characterization was obtained by Barnes and Rowlingson [15] as a subclass of the irrotational perfect fluid solutions with a purely electric Weyl tensor.

Invariant characterization 3 15]: A Szekeres-Szafron metric is characterized by the following conditions:

(i) It is a perfect fluid solution with a geodesic and irrotational unit velocity.

(ii) The Weyl tensor is purely electric and Petrov-Bel type D.

(iii) The shear tensor of the fluid has a double eigenvalue, and its associated eigenplane is the space-like principal plane of the Weyl tensor.

The above constraints are algebraic conditions for the Weyl tensor and first-order differential conditions for the Ricci tensor.

Takeno [16] referred to a characterization in "ideal form" when a spacetime is labeled by equations exclusively involving explicit concomitants of the metric tensor. He partially performed this type of analysis for the spherically symmetric spacetimes 16, 17], a result we attained in two recent papers [18, 19]. This kind of IDEAL characterization has also been achieved for other geometrically significant families of metrics and for physically relevant solutions of the Einstein equations [20-35]. The use of the appellation IDEAL (as an acronym) seems to be adequate because the conditions obtained are Intrinsic (depending only of the metric tensor), Deductive (not 
involving inductive or inferential methods or arguments), Explicit (expressing the solution non implicitly) and ALgorithmic (giving the solution as a flow chart with a finite number of steps). The IDEAL approach improves the previously known invariant characterizations since it can be achieved by using the current tensor calculus packages.

The IDEAL characterization of a metric is based on the Cartan historic results [36] and it can be useful, at least, in three fields of theoretical physics. First, it allows us to check whether a new solution to the Einstein equations is in fact already known. Consequently, it is also a method to study the metric equivalence problem, which is an alternative to the usual Cartan-Brans-Karlhede approach [37, 38]. Second, it is of interest in obtaining a fully algorithmic characterization of the initial data which correspond to a given solution. So, our IDEAL approaches to the Schwarzschild and Kerr solutions have been the starting point in several papers [39 41]. And third, it has been proposed as a fundamental tool in epistemic relativity for making gravimetry by using relativistic positioning systems and relativistic stereometric systems [2].

Section II is devoted to the first goal of this paper: provide an IDEAL labeling for the Szekeres-Szafron solutions. In spite of the wide range of invariant studies on these metrics their explicit expressions are not yet known. Our starting point is the invariant characterization 3 by Barnes and Rowlingson [15], and we must obtain the Ricci and Weyl concomitants that provide explicit expressions for the conditions (i), (ii) and (iii).

In his seminal paper, Rainich [43] stated the problem of obtaining the necessary and sufficient conditions for a metric to be a solution to the non-null Einstein-Maxwell equations, and he solved this problem in an IDEAL way. A similar study for the thermodynamic perfect fluid solutions was presented in Ref. [44]. By extension, we refer to a Rainich-like approach to a family of solutions when we give an IDEAL characterization of this family in terms of concomitants of the Ricci tensor (see for example Ref. 30]). Our second goal in this paper is to build the Rainich-like approach to the Szekeres-Szafron solutions, a task accomplished in Sec. III. This study can be useful in practical situations if we want to test if a metric defines a Szekeres-Szafron model by using exclusively the unit velocity $u$, the energy density $\rho$ and the pressure $p$. By using only hydrodynamic variables the Rainich-like approach also becomes useful from a conceptual point of view.

The Szekeres-Szafron metrics admit the canonical form $[1,2,[4,[5]$ :

$$
d s^{2}=-d t^{2}+e^{2 \alpha} d z^{2}+e^{2 \beta}\left(d x^{2}+d y^{2}\right),
$$

where the functions $\alpha=\alpha(t, z, x, y)$ and $\beta=\beta(t, z, x, y)$ are submitted to the field equations. These equations can be partially integrated by considering two classes, $\beta, z \neq 0$ (class I) and $\beta, z=0$ (class II) [1, 2]. In Sec. [V] we show that these coordinate conditions admit an invariant statement with a specific geometric interpre- tation, and we give them as explicit conditions for the Riemann tensor.

The Friedmann-Lemaitre-Robertson-Walker (FLRW) universes can be obtained from the Szekeres-Szafron spacetimes by means of a limiting procedure [1, 2]. An intermediate family of Szekeres-Szafron metrics, containing the FLRW universes, comprises the geodesic perfect fluid solutions admitting a three-dimensional group of isometries $O_{3}$ on space-like two-dimensional orbits $S_{2}$. In fact, the two-surfaces $t=$ const and $z=$ const of the metrics (1) of the Szekeres-Szafron type have constant curvature and, consequently, they admit a $G_{3}$. In Sec. $\mathrm{V}$ we study when this isometry group acts on the full spacetime and we offer the invariant and explicit conditions characterizing this family.

The invariant constraint distinguishing class I and class II Szekeres-Szafron metrics is a first-order differential condition (which is linear in the first derivatives) on the Riemann tensor. The condition characterizing the family with a $G_{3}$ on $S_{2}$ has similar properties. In Sec. VI we explore other possible first-order differential invariant conditions, and we comment on the invariant classes they define. These classes are also labeled by explicit expressions.

Some remarks are presented in Sec. VII on the interpretation of the Szekeres-Szafron models as a fluid in local thermal equilibrium. We also offer an intrinsic and explicit condition characterizing the thermodynamic Szekeres-Szafron spacetimes.

A discussion on the results is given in Sec. VIII We also offer one of all possible flow charts that can be built from our IDEAL characterizations, and which can easily be realized by using the current tensor calculus packages.

In Appendix $\mathrm{A}$ we give the expressions of the coordinate functions $\alpha$ and $\beta$ for the two classes I and II of the Szekeres-Szafron metrics, and also the expressions of some scalar invariants: pressure, energy density, Weyl eigenvalue and expansion. In Appendix B we prove some lemmas.

In this paper we work on an oriented spacetime with a metric tensor $g$ of signature $\{-,+,+,+\}$. The Weyl tensor $W$, the Ricci tensor $R$ and the scalar curvature $r$ are defined as given in Ref. [45]. For the metric product of two vectors, we write $(x, y)=g(x, y)$, and we put $x^{2}=g(x, x)$. The symbols $\nabla$ and $\nabla \cdot$ denote, respectively, the covariant derivative and the divergence operator. For a $(p+1)$-tensor $P$ and a $(q+1)$-tensor $Q, P \cdot Q$ denotes the $(p+q)$-tensor $(P \cdot Q)_{\bar{p} \bar{q}}=P_{\bar{p} \alpha} Q_{\bar{q}}^{\alpha}$, with $\bar{p}$ and $\bar{q}$ denoting multi-indices. For a two-tensor $T, T^{2}=T \cdot T$, $T(x)_{\alpha}=T_{\alpha \beta} x^{\beta}$ and $T(x, y)=T_{\alpha \beta} x^{\alpha} y^{\beta}$. For a vector field $x$ and a function $f$ we write $x(f)=x^{\alpha} \partial_{\alpha} f$.

\section{IDEAL CHARACTERIZATION OF THE SZEKERES-SZAFRON FAMILY OF METRICS}

In order to obtain an IDEAL labeling of the SzekeresSzafron solutions we must write the invariant conditions 
of characterization 3 in terms of explicit concomitants of the Ricci and Weyl tensors. Condition (i) consists of the algebraic constraints which guarantee the perfect fluid nature of the energy content, and the first-order differential ones which impose the geodesic and irrotational character of the fluid velocity.

The conditions for the Ricci tensor for a perfect fluid source were obtained years ago [4, [46]. Here we use a minor adaptation of a more recent version [19]:

Proposition 1 A spacetime is a perfect fluid solution if, and only if, the Ricci tensor $R$ satisfies

$$
\Gamma^{2}=\Gamma, \quad \Gamma(x, x)<0, \quad s \neq 0,
$$

where $x$ is any time-like vector, and

$$
\begin{gathered}
\Gamma \equiv \frac{1}{4} g-\frac{1}{s} N, \quad N \equiv R-\frac{r}{4} g, \\
s \equiv-2 \sqrt[3]{\frac{\operatorname{tr} N^{3}}{3}}, \quad r \equiv \operatorname{tr} R .
\end{gathered}
$$

Moreover, $\Gamma$ is the projector on the unit velocity $u, \Gamma=$ $-u \otimes u$, and the total energy $\rho$ and the pressure $p$ of the fluid are given by

$$
\rho=\frac{1}{4}(3 s+r), \quad p=\frac{1}{4}(s-r) .
$$

Now we must impose that the unit velocity has zero acceleration and rotation, $a=w=0$, and we must write these conditions in terms of the Ricci concomitant $\Gamma=-u \otimes u$. A straightforward calculation leads to the following.

Lemma 1 A unit time-like vector $u$ is geodesic and irrotational if, and only if, the projector $\Gamma=-u \otimes u$ satisfies

$$
\mathcal{A}=0, \quad \mathcal{A}_{\alpha \beta \mu} \equiv \nabla_{[\alpha} \Gamma_{\beta] \lambda} \Gamma_{\mu}^{\lambda} .
$$

Thus, we have explicit expressions for the invariant condition (i). Before studying condition (ii) we write the other kinematic coefficients of the velocity $u$ in terms of $\Gamma$. Again, a direct calculation allows us to obtain the following:

Lemma 2 If $u$ is a geodesic and irrotational unit timelike vector $(a=w=0)$ then

$$
\begin{array}{r}
\theta u=\Theta \equiv-\nabla \cdot \Gamma, \\
\sigma \otimes u=\Sigma \equiv\left[\frac{1}{3} \gamma \otimes(\nabla \cdot \Gamma)-\nabla \Gamma\right] \cdot \Gamma, \quad \gamma \equiv g-\Gamma .
\end{array}
$$

Condition (ii) in the invariant characterization 3 imposes algebraic constraints on the Weyl tensor. Nevertheless, if we calculate the electric and magnetic parts with respect to an observer $u$ comoving with the fluid, we obtain simpler explicit conditions involving both the Weyl tensor and the algebraic Ricci concomitant $\Gamma$. Indeed, remembering that a vanishing magnetic Weyl tensor implies Petrov-Bel types I, D or O, then, a simple scalar condition distinguishes the type $\mathrm{D}$ case. If we also take into account the definition of the electric and magnetic Weyl tensors, we obtain the following
Lemma 3 Let $W$ be the Weyl tensor and $\Gamma=-u \otimes u$ the projector on the unit time-like vector $u$. Then:

(i) The Weyl tensor $W$ is purely electric with respect the observer $u$ if, and only if,

$$
H=0, \quad H_{\alpha \beta} \equiv \Gamma^{\lambda \mu}(* W)_{\alpha \lambda \mu \beta} .
$$

(ii) The electric part $E$ of the Weyl tensor with respect to $u$ is

$$
E_{\alpha \beta} \equiv \Gamma^{\lambda \mu} W_{\alpha \lambda \mu \beta}
$$

(iii) If $H=0$, the Weyl tensor is of type $D$ or $O$ if, and only if,

$$
I^{3}=6 J^{2}, \quad I \equiv \operatorname{tr} E^{2}, \quad J \equiv \operatorname{tr} E^{3} .
$$

The conformally flat case (type $O$ ) occurs if, and only if, $E=0$.

Condition (iii) in the invariant characterization $3 \mathrm{im}-$ plies that the shear $\sigma$ and the electric Weyl tensor $E$ have a common eigenplane. But $E$ and $\sigma$ are traceless tensors and thus they are, necessarily, proportional. Conversely, this last condition implies that $\sigma$ admits an eigenplane provided that $E$ also admits this eigenplane. Moreover, we can use the Ricci concomitant $\Sigma$ defined in Eq. (8) to write this condition and we obtain the following

Lemma 4 If $E$ admits a double eigenvalue, then $\sigma$ admits the same eigenplane if, and only if,

$$
E_{\alpha \beta} \Sigma_{\lambda \mu \nu}=E_{\lambda \mu} \Sigma_{\alpha \beta \nu}
$$

where $\Sigma$ is given in Eq. (8).

Proposition 1] and lemmas 1, 2, 3] and 4 make explicit the three conditions in the invariant characterization 3. Thus, we have performed the IDEAL labeling of the Szekeres-Szafron solutions that we summarize in the following

Theorem 1 A metric tensor $g$ is a Szekeres-Szafron solution if, and only if, its Ricci tensor $R$ and Weyl tensor $W$ satisfy conditions (2), (6), (9), (11) and (12), where $\Gamma$ is given in Eq. (3), s in Eq. (4), $E$ in $E q$. (10), and $\Sigma$ in Eq. (8).

Included in the Szekeres-Szafron family of metrics characterized above are two notable limits: the nonexpanding solutions, which do not properly correspond to a cosmological model, and the FLRW universes, which occur in the conformally flat case or, equivalently, in the shear-free limit. Lemmas 2 and 3 lead to the following

Proposition 2 Let $g$ be a Szekeres-Szafron solution characterized in theorem 1 and let $\Theta$ and $E$ and be the Riemann concomitants defined in Eqs.(7) and (10). Then:

(i) The metric $g$ defines a cosmological model with a nonvanishing expansion if, and only if, $\Theta \neq 0$.

(ii) The metric $g$ defines a FLRW universe if, and only if, $\Theta \neq 0$ and $E=0$. 


\section{CHARACTERIZATION IN TERMS OF THE RICCI TENSOR: RAINICH-LIKE APPROACH}

In order to obtain an IDEAL labeling of the SzekeresSzafron metrics solely in terms of the Ricci tensor we could start from the invariant characterization 2 which imposes third-order differential conditions on the Ricci tensor, and then we should get explicit expressions for them in order to perform a Rainich-like approach. Nevertheless, we will prove here that starting from our IDEAL labeling presented in the previous section and based on characterization 3, we acquire a Rainich-like approach which imposes second-order differential conditions on the Ricci tensor.

This approach is possible because the Ricci identities for the unit velocity of the fluid give us the electric and magnetic Weyl tensors in terms of second-order Ricci concomitants. Indeed, for a geodesic an irrotational unit velocity, the Ricci identities imply [45]

$$
\begin{array}{r}
E=-\frac{2}{3} \theta \sigma-\dot{\sigma}-\sigma^{2}+\frac{1}{3} \operatorname{tr} \sigma^{2} \gamma, \\
H=\operatorname{curl} \sigma .
\end{array}
$$

where a dot denotes the covariant directional derivative along the unit velocity $u$.

Identity (14) shows that the algebraic invariant $H$ given in Eq. (9), which depends on the Weyl and Ricci tensors, is equal to a first-order differential invariant depending solely on the Ricci tensor. We can write this Ricci invariant, which we denote $\mathcal{H}$, in terms of $\Sigma$, and we obtain the following

Lemma 5 Let $u$ be a geodesic and irrotational unit vector. The magnetic part of the Weyl tensor with respect to $u$ vanishes if, and only if,

$$
\mathcal{H}=0, \quad \mathcal{H}^{\alpha \beta} \equiv \eta^{\lambda \mu \nu(\alpha} \nabla_{\lambda} \Sigma_{\mu \nu}^{\beta)},
$$

where $\Sigma$ is given in Eq. (8) and $\Gamma=-u \otimes u$.

Similarly, identity (13) shows that the invariant $E$ given in Eq. (10), which depends algebraically on the Weyl and Ricci tensors, is equal to a second-order differential invariant depending solely on the Ricci tensor. We could write this Ricci invariant in terms of $\Theta$ and $\Sigma$, and impose on it the constraints (11) and (12). This procedure leads to tangled conditions with nonlinear secondorder terms. Nevertheless, we can obtain simpler conditions by imposing on the shear $\sigma$ the condition of having a double eigenvalue. A straightforward calculation allows us to write this condition in terms of $\Sigma$ and we get the following

Lemma 6 A geodesic and irrotational unit vector $u$ has a shear with a double eigenvalue if, and only if,

$$
6 \mathcal{S}^{2}=S^{3}, \mathcal{S}_{\alpha} \equiv \Sigma_{\lambda \beta \gamma} \Sigma_{\mu}^{\beta \gamma} \Sigma^{\lambda \mu}{ }_{\alpha}, \quad S \equiv \Sigma_{\lambda \mu \nu} \Sigma^{\lambda \mu \nu} .
$$

where $\Sigma$ is given in Eq. (8) and $\Gamma=-u \otimes u$.
On the other hand, we must impose the proportionality of $\sigma$ and $E$. From Eq. (13) this condition is equivalent to the proportionality of $\sigma$ and $\dot{\sigma}$. If we write this condition in terms of $\Sigma$ we obtain the following

Lemma 7 For a geodesic and irrotational unit vector $u$, the shear $\sigma$ and its derivative $\dot{\sigma}$ are proportional tensors if, and only if,

$$
\nabla_{\nu} \Sigma_{\alpha \beta}^{\nu} \Sigma_{\lambda \mu \gamma}=\nabla_{\nu} \Sigma_{\lambda \mu}^{\nu} \Sigma_{\alpha \beta \gamma} .
$$

where $\Sigma$ is given in $E q$. (8) and $\Gamma=-u \otimes u$.

Proposition 1 and lemmas 5, 6 and7 7 allow us to replace conditions (9), (11) and (12) in theorem 11 with conditions (15), (16) and (17). This way, the first-order conditions on the Weyl and Ricci tensors can be replaced by secondorder ones involving only the Ricci tensor. Consequently, we acquire the following Rainich-like characterization:

Theorem 2 A metric tensor $g$ is a Szekeres-Szafron solution if, and only if, its Ricci tensor $R$ satisfies conditions (2), (6), (15), (16) and (17), where $\Gamma$ is given in $E q$. (3), $s$ in $E q$. (4), and $\Sigma$ in Eq. (8).

The FLRW universes limit also admits a characterization in terms of the Ricci tensor:

Proposition 3 A Szekeres-Szafron solution characterized in theorem 2 becomes a FLRW universe if, and only if, $\Theta \neq 0$ and $\Sigma=0$, where $\Theta$ and $\Sigma$ are given in Eqs. (7) and (8).

\section{IDEAL LABELING OF BOTH FAMILIES OF SZEKERES-SZAFRON METRICS}

Szafron and Collins 13 showed that the coordinate condition $\beta, z=0$, which defines the class II of the Szekeres-Szafron metrics, admits an invariant statement for the strict Szekeres-Szafron (sSS) metrics, that is, when $\sigma \neq 0$ (the spacetime is not a FLRW universe). Here, to provide an interpretation of this invariant condition and to obtain an explicit expression of it in terms of the curvature tensor, we analyze the geometric properties of the unitary eigenvector $b$ associated with the simple eigenvalue of the electric Weyl tensor.

In the canonical coordinate system (1) $b$ and its covariant derivative take the forms

$$
\begin{aligned}
& b=e^{\alpha} d z, \quad \nabla b=b \otimes a_{b}+\frac{1}{2} \theta_{b} h, \quad h \equiv \gamma-b \otimes b, \\
& a_{b} \equiv \nabla_{b} b=\dot{\alpha} u-h(d \alpha), \quad \theta_{b} \equiv \nabla \cdot b=2 e^{-\alpha} \beta_{z} .
\end{aligned}
$$

For a function $f, \dot{f}=u(f)=u^{\alpha} \partial_{\alpha} f=f, t$. Note that $h$ is the projector on the space-like principal plane of the Weyl tensor, and $a_{v}$ and $\theta_{v}$ are, respectively, the acceleration and the expansion of the eigenvector $b$. Equation (18) shows that $b$ is expansion-free if, and only if, it is shearfree. Then, Eq. (19) implies the following 
Lemma 8 For the strict Szekeres-Szafron solutions we have the following three equivalent conditions:

(i) The metric is of class II $(\beta, z=0)$.

(ii) The simple eigenvector of the electric Weyl tensor is expansion-free.

(ii) The simple eigenvector of the electric Weyl tensor is shear-free.

On the other hand, the projectors on the direction defined by the simple eigenvector, $B=b \otimes b$, and on the space-like principal plane, $h$, can be obtained as

$$
B \equiv \frac{1}{3}\left(\frac{2}{\omega} E+\gamma\right), \quad h \equiv \gamma-B, \quad \omega \equiv \frac{2 J}{I},
$$

where $\gamma, E, I$ and $J$ are given in Eqs. (8), (10) and (11). Note that $\omega$ is the simple Weyl eigenvalue. Finally, if we write condition $\theta_{b}=0$ in terms of $B$, and we take into account lemma 8, we obtain the following

Proposition 4 Let $g$ be a Szekeres-Szafron solution characterized in theorem 1. Then, if $E \neq 0, g$ is of class II if, and only if, the Ricci and Weyl tensors satisfy

$$
B(\nabla \cdot B)=0,
$$

where $B$ is given in Eq. (20).

In order to obtain a Rainich-like labeling of the Szekeres-Szafron metrics of class I and class II, we must write the condition $\theta_{b}=0$ only in terms of the Ricci tensor. We know that $b$ is also the eigenvector of $\sigma$ associated with the simple eigenvalue. Then, from the expressions (8) and (16) we obtain

$$
\begin{array}{r}
b \otimes b \otimes u=\mathcal{B} \equiv-\frac{1}{\sqrt{-\mathcal{S}^{2}}}[S \Sigma+\gamma \otimes \mathcal{S}], \\
h_{\alpha \beta}=\gamma_{\alpha \beta}+\mathcal{B}_{\alpha}^{\lambda \mu} \mathcal{B}_{\beta \lambda \mu},
\end{array}
$$

where $\Sigma, \gamma, \mathcal{S}$ and $S$ are given in Eqs. (8) and (16). Finally, if we write the condition $\theta_{b}=0$ in terms of $\mathcal{B}$, and we take into account lemma 8, we obtain the following

Proposition 5 Let $g$ be a Szekeres-Szafron solution characterized in theorem 2. Then, if $\Sigma \neq 0, g$ is of class II if, and only if, the Ricci tensor satisfies

$$
\mathcal{B}_{\alpha \lambda \mu}(\nabla \cdot \mathcal{B})^{\lambda \mu}=0,
$$

where $\mathcal{B}$ is given in $E q$. (22).

\section{INVARIANT AND IDEAL LABELING OF THE SZEKERES-SZAFRON METRICS ADMITTING A $G_{3}$ ON $S_{2}$.}

It is known [2] that the two-surfaces $t=$ const, $z=$ const of the Szekeres-Szafron spacetimes have constant curvature, that is, they admit a maximal group of isometries $G_{3}$. From the coordinate expressions given in Appendix A we can analyze when this group is a group of isometries of the full spacetime. We have the following result:
Lemma 9 For a strict Szekeres-Szafron solution the three following conditions are equivalent:

(i) It admits a three-dimensional group $G_{3}$ on twodimensional space-like orbits $S_{2}$.

(ii) $\alpha,{ }_{x}=\alpha,{ }_{y}=0(h(d \alpha)=0)$.

(iii) $\nu, z x=\nu, z y=0$ for class $I(\beta, z \neq 0)$, or $P,_{x}=P,_{y}=$ 0 for class $I I(\beta, z=0)$.

From Eq. (11) and expressions in Appendix A, it is evident that (i) implies (ii), and (ii) implies (iii). It is also trivial that (iii) leads to (i) for the class-II Szekeres-Szafron metrics. For class I, condition (iii) implies $(\ln \mathrm{S})_{x z}=$ $(\ln \mathrm{S})_{y z}=0$, and then the functions $U(z), V_{1}(z), V_{2}(z)$ and $W(z)$ in the expression (A2) of $\mathrm{S}(z, x, y)$ differ by a constant. Then, a linear change in the $(x, y)$ coordinates leads to $\mathrm{S}(z, x, y)=U(z) C(x, y)$, where $C(x, y)$ takes the form (A9), and then (i) follows.

The invariant condition $h\left(a_{b}\right)=0$ is equivalent to $h(d \alpha)=0$ as a consequence of Eq. (19). Thus the lemma above implies that this invariant condition characterizes the sSS metrics admitting a three-dimensional group of isometries $G_{3}$ with space-like two-dimensional orbits $S_{2}$. Furthermore, the null Weyl principal directions are $\ell_{ \pm}=u \pm b=-d t \pm \alpha d z$, and a straightforward calculation shows that they are tangent to null geodesics if, and only if, $h(d \alpha)=0$. Moreover, condition $h\left(a_{b}\right)=0$ can be written in terms of one of the Riemann concomitants $B$ and $\mathcal{B}$ defined in Eqs. (20) and (22), respectively. Consequently, we have the following

Proposition 6 For a strict Szekeres-Szafron metric the following conditions are equivalent:

(i) It admits a three-dimensional group $G_{3}$ on twodimensional space-like orbits $S_{2}$.

(ii) The null Weyl principal directions define geodesic congruences.

(iii) $h\left(a_{b}\right)=0$, where $a_{b}$ is the acceleration of the simple Weyl eigenvector $b$.

(iv) $h(\nabla \cdot B)=0$, where $h$ and $B$ are given in $E q$. (20).

(v) $h \cdot(\nabla \cdot \mathcal{B})=0$, where $\mathcal{B}$ and $h$ are given in Eqs. (22) and (23) respectively.

Condition (ii) was introduced by Wainwright [47] in classifying the type D perfect fluid solutions, showing that if one of the two null principal directions is geodesic then both are. Moreover, he proved [12] that (ii) is a sufficient condition for the existence of a $G_{3}$ on $S_{2}$ in the case of a dust Szekeres metric.

Our proposition 6] states that this condition is also a necessary one, and we extend the result to the full set of the sSS metrics. Conditions (ii) and (iii) are two equivalent invariant conditions which characterize the SzekeresSzafron metrics admitting a $G_{3}$ on $S_{2}$. Furthermore, condition (iv) is explicit in both the Weyl and the Ricci tensor, and (v) is explicit in the Ricci tensor. 
Proposition 6 implies that the sSS metrics with $h\left(a_{b}\right)=$ 0 are perfect fluid solutions with geodesic velocity admitting a $G_{3}$ on $S_{2}$. Conversely, the existence of this group of isometries implies that the spacetime is Petrov-Bel type $\mathrm{D}$, the space-like principal plane being tangent to the group orbits and an eigenplane of the shear. Also the fluid is irrotational. Consequently, under the geodesic constraint, all the conditions in the invariant characterization 3 of the Szekeres-Szafron metrics hold. Thus, we have the following

Proposition 7 The Szekeres-Szafron metrics with $h\left(a_{b}\right)=0$ are the perfect fluid solutions with geodesic velocity admitting a group of isometries $G_{3}$ on orbits $S_{2}$.

In the next section we will prove that there are other invariant conditions, which are also linear in the first derivatives of the Riemann tensor, that either imply or are equivalent to the existence of a $G_{3}$ on $S_{2}$. Now we analyze the second-order condition $h(d \theta)=0$. From Eq. (A6) (respectively, Eq. (A14)) of the expansion of the fluid of a sSS metrics of class I (respectively, class II), we obtain that $h(d \theta)=0$ implies $\left(\dot{\phi} \phi_{z}-\phi \dot{\phi}, z\right) h\left(d \nu_{z}\right)=0$ (respectively, $(\dot{\phi} \lambda-\phi \dot{\phi} \lambda, z) h(d P)=0)$. The factor $\dot{\phi} \phi_{z}-\phi \dot{\phi}, z$ (respectively, $\dot{\phi} \lambda-\phi \dot{\phi} \lambda, z$ ) only vanishes in the FLRW limit. Thus, for the $\mathrm{sSS}$ metrics $h(d \theta)=0$ implies that $\nu, z x=\nu, z y=0$ (respectively, $P_{x}=P_{y}=0$ ) and, as a consequence of lemma 9, the spacetime admits a $G_{3}$ on $O_{2}$. Conversely, this condition implies $h(d \theta)=0$ since any Riemann scalar is invariant by the group. Thus, we can state the following

Proposition 8 A strict Szekeres-Szafron metric admits an isometry group $G_{3}$ on orbits $\mathrm{O}_{2}$ if, and only if, the invariant condition $h(d \theta)=0$ holds.

The explicit expression for this constraint is $h \cdot \nabla \Theta \cdot \Gamma=0$, where $\Gamma$ is given in Eq. (3), $\Theta$ in Eq. (7), and $h$ in Eq. (20).

As a consequence of the proposition above, the SzekeresSzafron solutions with homogeneous expansion, $d \theta \wedge u=$ 0 , admit a $G_{3}$ on $S_{2}$.

\section{OTHER SZEKERES-SZAFRON SUBCLASSES DEFINED BY FIRST-ORDER DIFFERENTIAL INVARIANT CONDITIONS}

The invariant tensor $B$ defined in Eq. (20) is an algebraic Riemann invariant. Consequently, constraint (21) that characterizes the sSS metrics of class II is a firstorder differential condition, which is linear in the first derivatives. The constraint $h(\nabla \cdot B)=0$ that characterizes the sSS admitting a $G_{3}$ on $S_{2}$ has similar qualities. Any Riemann invariant with analogous differential properties will generate a classification of these metrics. In order to index all these invariants let us notice first the algebraic Riemann invariants: unit velocity $u$, energy density $\rho$, pressure $p$, simple Weyl eigenvalue $\omega$, and simple associated eigenvector $b$.
Then, the first-order differential invariants (which are linear in the first derivatives) are defined by the kinematic coefficients of the invariant vectors $u$ and $b$, and the projection on $u, b$ and $h$ (projector on the space-like eigenplane) of the gradient of the scalar invariants $\rho, p$ and $\omega$. For the Szekeres-Szafron metrics some of these invariants identically vanish. Moreover, $\theta_{b}=0$ if, and only if, $\sigma_{b}=0$ (lemma 8), and then it is enough to consider any of these two invariants. Furthermore, the acceleration $a_{b}$ can be projected on $u$ and on the space-like Weyl principal plane. Now we analyze the classification induced by each of these differential invariants.

\section{A. Classes defined by invariants associated with the unit velocity $u$}

The only nonvanishing coefficients are the shear $\sigma$ and the expansion $\theta$. We know that the first-order condition $\sigma=0$ is equivalent to the algebraic one $E=0$, and it leads to the FLRW universes. In this case the algebraic invariants $b$ and $\omega$ are not defined. Otherwise, when $\sigma \neq$ 0 , we have the sSS metrics, a set where the other firstorder invariants induce a classification that we consider in the following subsections.

Moreover, as a consequence of the conservation equation, $\dot{\rho}+(\rho+p) \theta=0, \theta=0$ is a constraint equivalent to $u(d \rho)=\dot{\rho}=0$, a condition that we analyze below for the sSS metrics.

Note that both conditions, $\sigma=0$ and $\theta=0$ admit an explicit expression in terms of the Ricci invariants defined in Eqs. (8) and (7): $\Sigma=0$ and $\Theta=0$.

\section{B. Classes defined by invariants associated with the simple eigenvector $b$}

Now we can consider the expansion $\theta_{b}$ and the projections $\left(a_{b}, u\right), h\left(a_{b}\right)$ of the acceleration $a_{b}$ of the vector $b$.

In lemma 8 of Sec. IV we have shown that the condition $\theta_{b}=0$ characterizes the sSS metrics of class II, and we have also presented ideal statements for this condition (propositions 4 and 5).

Furthermore, the analysis presented in the previous section shows that the condition $h\left(a_{b}\right)=0$ characterizes the sSS metrics admitting an isometry group $G_{3}$ on orbits $S_{2}$, and we have presented ideal statements for this condition (proposition 6).

Finally, the invariant condition $\left(a_{b}, u\right)=0$ is equivalent to $\alpha, t=0$ as a consequence of Eq. (19). For sSS metrics of class I (respectively, class II), Eqs. (A1) and (A2) (respectively, Eqs. (A7) and (A10) imply that this condition leads to $\dot{\phi},{ }_{z}+\dot{\phi} \nu,{ }_{z}=0$ (respectively, $\dot{\lambda}+P \dot{\phi}=0$ ) and then $\dot{\phi} \nu, z x=\dot{\phi} \nu, z y=0$ (respectively, $\dot{\phi} P,_{x}=\dot{\phi} P,_{y}=0$ ). Thus, either $\dot{\phi}=0$ (respectively, $\dot{\phi}=\dot{\lambda}=0$ ) and the metric is static, or $\nu, z x=\nu, z y=0$ (respectively, $P,_{x}=P,_{y}=0$ ) and the spacetime admits a 
$G_{3}$ on $S_{2}$ (see lemma 9). Note that the static condition is not possible in the sSS metrics. On the other hand, we can write the invariant condition $\left(a_{b}, u\right)=0$ in terms of the explicit Riemann concomitants $B$ and $\mathcal{B}$. Thus, we obtain the following

Proposition 9 For a strict Szekeres-Szafron metric the following conditions are equivalent:

(i) $\left(a_{b}, u\right)=0$.

(ii) $\Gamma(\nabla \cdot B)=0$ with $B$ given in Eq. (20), and $\Gamma$ in Eq. (3).

(iii) $\Gamma \cdot(\nabla \cdot \mathcal{B})=0$ with $\mathcal{B}$ given in Eq. (22) and $\Gamma$ in $E q$. (3).

Moreover, any of these conditions implies $h\left(a_{b}\right)=0$, that $i s$, the spacetime admits necessarily an isometry group $G_{3}$ on orbits $S_{2}$.

Note that condition (ii) is an explicit expression of (i) in terms of the Ricci and Weyl tensors, and (iii) is its explicit expression only in terms of the Ricci tensor.

\section{Classes defined by invariants associated with the pressure $p$}

For the sSS metrics we have $d p \wedge u=0$, and then the sole scalar $u(p)=\dot{p}$ can be considered. If it vanishes, $u(p)=0$, we have a constant pressure. This class, which includes the Szekeres dust solutions, has been widely studied by several authors (see Refs. [1, 2, 10, 11] and references therein).

As a consequence of Eq. (5), condition $u(p)=0$ admits an explicit expression in terms of Ricci invariants: $d r=$ $d s$, where $r$ and $s$ are given in Eq. (4).

\section{Classes defined by invariants associated with the simple Weyl eigenvalue $\omega$}

Now we have the invariants defined by the projections $u(\omega), b(\omega), h(d \omega)$ of the gradient $d \omega$ of the scalar $\omega$.

First we analyze the invariant condition $h(d \omega)=0$. From Eq. (A5) (respectively, Eq. (A13) of the Weyl eigenvalue of a sSS metrics of class I (respectively, class II) we obtain $h(d \omega)=-\omega \frac{\phi}{\phi_{z}+\phi \nu_{z}} h\left(d \nu_{z}\right)$ (respectively, $\left.h(d \omega)=-\omega \frac{\phi}{\lambda+\phi P} h(d P)\right)$. Thus $h(d \omega)=0$ implies that $\nu, z x=\nu,{ }_{z y}=0$ (respectively, $P_{x}=P_{y}=0$ ) and, as a consequence of lemma [9] the spacetime admits a $G_{3}$ on $\mathrm{O}_{2}$. Conversely, this condition implies $h(d \omega)=0$ since any Riemann scalar is invariant by the group. Thus, we can state the following

Proposition 10 A strict Szekeres-Szafron metric admits an isometry group $G_{3}$ on orbits $\mathrm{O}_{2}$ if, and only if, the invariant condition $h(d \omega)=0$ holds.

The explicit expressions of the involved Riemann invariants $h$ and $\omega$ are given in Eq. (20).
Second we analyze the invariant condition $u(\omega)=0$, that is, $\dot{\omega}=0$. From Eq. A5 (respectively, Eq. A13) of the Weyl eigenvalue of a sSS metric of class I (respectively, class II) we obtain $h(d \dot{\omega})=-\omega \partial_{t}\left(\frac{\phi}{\phi_{z}+\phi \nu_{z}}\right) h\left(d \nu_{z}\right)$ (respectively, $\left.h(d \dot{\omega})=-\omega \partial_{t}\left(\frac{\phi}{\lambda+\phi P}\right) h(d P)\right)$. The factor $\partial_{t}\left(\frac{\phi}{\phi_{z}+\phi \nu_{z}}\right)$ (respectively, $\partial_{t}\left(\frac{\phi}{\lambda+\phi P}\right)$ ) only vanishes in the FLRW limit. Thus, for the sSS metrics $u(\omega)=0$ implies that $\nu, z x=\nu, z y=0$ (respectively, $P_{x}=P_{y}=0$ ) and, as a consequence of lemma 9. the spacetime admits a $G_{3}$ on $O_{2}$. Thus, we can state the following

Proposition 11 For a strict Szekeres-Szafron metric the invariant condition $u(\omega)=0$ implies $h(d \omega)=0$, that is, the spacetime admits an isometry group $G_{3}$ on orbits $\mathrm{O}_{2}$. Moreover, the condition $u(\omega)=0$ admits the explicit statement $\Gamma(d \omega)=0$, where $\Gamma$ is given in Eq. (3) and $\omega$ in Eq. (20).

Now we need to analyze the invariant condition $b(\omega)=$ 0 , that is, $\omega, z=0$. For a sSS metric of class I, lemma 10 in Appendix B implies that $\nu_{z x}=\nu_{z y}=0$ and, as a consequence of lemma 9, the spacetime admits a $G_{3}$ on $\mathrm{O}_{2}$. For a sSS metric of class II, lemma 11 in Appendix $\mathrm{B}$ implies that $\xi=\partial_{z}$ is a Killing vector. Thus, we have the following

Proposition 12 For a strict class-I Szekeres-Szafron metric the invariant condition $b(\omega)=0$ implies $h(d \omega)=$ 0 , that is, the spacetime admits an isometry group $G_{3}$ on orbits $\mathrm{O}_{2}$.

For a strict class-II Szekeres-Szafron metric the invariant condition $b(\omega)=0$ implies that the Weyl eigenvector $b$ determines a Killing vector direction.

Moreover, the condition $b(\omega)=0$ admits the explicit statement $B(d \omega)=0$, where $B$ and $\omega$ are given in Eq. (20).

\section{E. Classes defined by invariants associated with the energy density $\rho$}

Now we have the projections $u(\rho), b(\rho)$ and $h(d \rho)$ of the gradient $d \rho$ of the the scalar $\rho$. From the expressions of the pressure $p$, the energy density $\rho$ and the Weyl eigenvalue $\omega$ given in Appendix $\mathrm{A}$ we obtain

$$
\frac{1}{3} \rho+p+2 \omega=-2 \frac{\ddot{\phi}}{\phi} .
$$

From this relation we have that $h(d \rho)=0$ if, and only if, $h(d \omega)=0$. Consequently, proposition 10 applies and we can state the following

Proposition 13 A strict Szekeres-Szafron metric admits an isometry group $G_{3}$ on orbits $\mathrm{O}_{2}$ if, and only if, the invariant condition $h(d \rho)=0$ holds.

The explicit expressions of the involved Riemann invariants $h$ and $\rho$ are given in Eqs. (20) and (5), respectively. 
Now we analyze the invariant condition $u(\rho)=0$, that is, $\dot{\rho}=0$ or, equivalently $\theta=0$. From Eq. (A6) (respectively, Eq. (A14) ) of the expansion of a sSS metrics of class I (respectively, class II) we obtain $\phi \dot{\phi},{ }_{r}+3 \phi \dot{\phi} \nu_{, r}+2 \dot{\phi} \phi,{ }_{r}=0$ (respectively, $\phi \dot{\lambda}+3 \phi \dot{\phi} P+$ $2 \dot{\phi} \lambda=0)$ and, differentiating with respect to $x$ and $y$, we have that $\nu, z x=\nu,_{z y}=0$ (respectively, $P_{x}=P_{y}=0$ ) and, as a consequence of lemma 9, the spacetime admits a $G_{3}$ on $O_{2}$. Thus, we can state the following

Proposition 14 For a strict Szekeres-Szafron metric the invariant condition $u(\rho)=0$ implies $h(d \rho)=0$, that $i s$, the spacetime admits an isometry group $G_{3}$ on orbits $\mathrm{O}_{2}$. Moreover, the condition $u(\rho)=0$ admits the explicit statement $\Gamma(d \rho)=0$, where $\Gamma$ is given in $E q$. (3) and $\rho$ in Eq. (5).

In the case of the invariant condition $b(\rho)=0$, that is $\rho, z=0$, we must distinguish between class I and class II metrics. In the second case $\phi=\phi(t)$ and then Eq. (25) implies that $b(\rho)=0$ if, and only if, $b(\omega)=0$, and then proposition 12 applies and the spacetime admits a Killing vector which is parallel to $b$. In the first case, for metrics of class I, the spacetime is a FLRW universe as a consequence of lemma 12 in Appendix B. Thus, we have the following

Proposition 15 Strict Szekeres-Szafron metrics of type I fulfilling the invariant condition $b(\rho)=0$ do not exist. For a strict Szekeres-Szafron metric of type II the invariant condition $b(\rho)=0$ implies that the Weyl eigenvector $b$ determines a Killing vector direction.

Moreover, the condition $b(\rho)=0$ admits the explicit statement $B(d \rho)=0$, where $B$ and $\rho$ are given in Eqs. (20) and (5), respectively.

\section{ON THE THERMODYNAMIC SZEKERES-SZAFRON SOLUTIONS}

A relevant step in studying perfect fluid solutions is to analyze their interpretation as reasonable physical media. The Plebański [48] energy conditions are necessary algebraic conditions for physical reality and, in the perfect fluid case, they state $-\rho<p \leq \rho$. The determination of the spacetime regions where these constraints hold is a basic query in analyzing a given perfect fluid solution.

Furthermore, if we want the solution to describe a perfect fluid in local thermal equilibrium we must impose complementary constraints. A necessary condition for the fluid to admit a thermodynamic scheme is that a function $n$ exists such that [49]

$$
\dot{n}+n \theta=0, \quad d n \wedge d p \wedge d \rho=0 .
$$

Then, the function of state $n=n(\rho, p)$ is the conserved matter density of the fluid.

It is worth remarking that Eq. (26) is not an intrinsic condition on a perfect energy tensor $T \equiv(u, \rho, p)$ in order to represent the energetic evolution of a perfect fluid in local thermal equilibrium. Indeed, it involves the function $n$, which is not defined by $T$. In Ref. 44] (see also Ref. [49]) we presented an intrinsic and explicit condition: a nonisoenergetic $(\dot{\rho} \neq 0)$ perfect energy tensor $T$ evolves in local thermal equilibrium if, and only if, the hydrodynamic variables $(u, \rho, p)$ fulfill

$$
d \chi \wedge d p \wedge d \rho=0, \quad \chi \equiv \frac{\dot{p}}{\dot{\rho}} .
$$

Then the indicatrix of the local thermal equilibrium $\chi$ is a function of state, $\chi=\chi(\rho, p)$, which represents the square of the speed of sound [49]. This IDEAL characterization of local thermal equilibrium enabled us to construct a Rainich-like theory for the thermodynamic perfect fluids [44]. To get this, it is enough to write condition (27) in terms of the Ricci tensor and to add it to the conditions in proposition 10 44, 19].

In the case of the Szekeres-Szafron spacetimes this thermodynamic condition admits an equivalent and simpler expression. Indeed as $p$ is a function of $t$, we have $d p \wedge u=d \dot{p} \wedge u=0$. Then, Eq. (27) is equivalent to $d \dot{\rho} \wedge d \rho \wedge u=0$. Also $\dot{\rho}$ can be substituted by $\theta$ because of the conservation equation $\dot{\rho}+(\rho+p) \theta=0$. Thus, Eq. (27) becomes

$$
d \theta \wedge d \rho \wedge u=0 .
$$

Moreover, we can substitute $\rho$ by any of the Ricci scalar invariants, $r$ and $s$, defined in Eq. (44). The resulting constraint can be written in terms of $\Gamma$ and $\Theta$, and we obtain the following

Proposition 16 Let $g$ be a Szekeres-Szafron solution characterized in theorem 1 or in theorem $\mathbb{2}$. Then, the solution represents a perfect fluid in local thermal equilibrium if, and only if, the Ricci tensor satisfies

$$
\mathcal{T}=0, \quad \mathcal{T}^{\alpha} \equiv \eta^{\alpha \lambda \mu \nu} \Gamma_{\lambda}^{\beta} \partial_{\mu} r \nabla_{\nu} \Theta_{\beta} .
$$

where $\Gamma$ is given in Eq. (3), $r$ in $E q$. (4) and $\Theta$ in $E q$. (7).

The above IDEAL labeling of the thermodynamic Szekeres-Szafron metrics is mainly relevant from a conceptual point of view. Nevertheless, in order to obtain physically realistic models there are still many steps to take: (i) obtain the complementary constraints that the thermodynamic condition imposes on the metric coordinate functions $\alpha$ and $\beta$; (ii) determine for these thermodynamic models the expression $\chi(\rho, p)$ of the indicatrix function; (iii) solve, for this indicatrix function, the inverse problem of determining the thermodynamic scheme that defines the thermodynamic properties of the fluid.

Starting from the conditions (26) Krasiński et al. [50] proved that if a class I Szekeres-Szafron metric admits a thermodynamic scheme then, necessarily, it admits symmetries. Nevertheless, there are thermodynamic Szekeres-Szafron solutions of class II without symmetries 
[50]. The result of Krasiński et al. 50] concerning class I can be easily found following Eq. (28). Indeed, if we impose Eq. (28) taking into account Eqs. (A12) and (A14), we obtain:

$$
\dot{Q} F,{ }_{z} h(d \nu)=0, \quad F \equiv \frac{\ddot{\phi}}{\phi},
$$

where $Q$ is given in Eq. (B5). The constraint $F,_{z}=0$ leads to the FLRW limit. Then, from condition (30), lemma 9 and lemma 13 in Appendix B, we recover the Krasiński et al. result [50] (see also Ref. [2]):

Proposition 17 A strict Szekeres-Szafron solution of type I represents a perfect fluid in local thermal equilibrium if, and only if, the spacetime admits a group of isometries $G_{3}$ on orbits $S_{2}$, that is, the metric satisfies any of the equivalent conditions in proposition 6 .

All the above-quoted results contribute to the first step (i) in looking for realistic models. Years ago we presented some preliminary results on the inverse problem for type II sSS metrics [51]. The exhaustive analyses of this subject, which require the results of the recent paper [49], is an ongoing work that will be considered elsewhere.

\section{DISCUSSION}

Starting from the invariant characterization by Barnes and Rowlingson [15], in this paper we have presented two IDEAL (intrinsic, deductive, explicit and algorithmic) characterizations of the Szekeres-Szafron universes. The first one is of the lowest order (first derivatives in the Riemann tensor) and involves both the Weyl and the Ricci tensors (Sec. III). The second one constitutes a Rainich-like approach and it requires second-order conditions solely in terms of the Ricci tensor (Sec. III).

It is worth remarking that the conditions that we found in the above-cited characterizations involve algebraic and differential concomitants of invariant vectors, like the velocity $u$ and the simple Weyl eigenvector $b$. These invariant vectors can be explicitly obtained in terms of the associated projectors, which are concomitants of the Riemann tensor. For example, $u=-[-\Gamma(x, x)]^{-\frac{1}{2}} \Gamma(x)$, where $\Gamma$ is given in (3) and $x$ is any time-like vector. Then, when we impose any condition on $u$, this condition involves an arbitrary vector $x$. In order to prevent this $x$ from appearing in the characterization equations, we have opted to use concomitants of the projector $\Gamma$. Thus, for example, we worked with $\Sigma$ and $\Theta$ instead of with $\sigma$ and $\theta$. A similar situation occurs with the simple Weyl eigenvector $b$ and its associated projector $B$.

We have also explicitly labeled some significant subfamilies of the Szekeres-Szafron solutions: the sSS metrics of class I and class II, which appear in a natural way when integrating the field equations (Sec. IV), and the Szekeres-Szafron metrics admitting a three-dimensional group of isometries $G_{3}$ on space-like two-dimensional orbits $S_{2}$ (Sec. $\left.\mathrm{V}\right)$. It is worth remarking that the metrics of this last class are the geodesic perfect fluid solutions with these symmetries (proposition 17). Moreover, there are several equivalent invariant conditions labeling this class, and all of them impose that the projection of an invariant vector on the space-like principal plane (tangent to the orbits group) vanishes (propositions 6, 8, 10 and 13).

The analysis of the subfamilies of the Szekeres-Szafron metrics defined by first-order invariant conditions (linear in the first derivatives) shows that a few significant classes can be considered (Sec. VI). We have the type I and type II sSS metrics, and those admitting a $G_{3}$ on $S_{2}$, which we have quoted in the paragraph above as well as the solutions with constant pressure, which generalize the dust solutions by Szekeres. The other defined subfamilies necessarily admit symmetries: either a $G_{3}$ on $S_{2}$, or a $G_{1}$ with the simple Weyl eigenvector $b$ tangent to the orbits.

We have also given an IDEAL labeling of the SzekeresSzafron solutions that can be interpreted as a perfect fluid in local thermal equilibrium (Sec. VII).

It is worth noting the powerful advantages of an IDEAL characterization over the previous known invariant ones. Indeed, the deductive, explicit and algorithmic qualities allow us to built a flow chart with a finite number of steps and to implement it easily by using the current tensor calculus packages.

The diagram below presents one of all possible flow charts that can be built from our results. It allows us to distinguish the perfect fluid solutions, the SzekeresSzafron metrics (SS), the FLRW universes and the strict Szekeres-Szafron metrics of classes I (sSS-I) and II (sSSII). We make use of the Ricci and Weyl concomitants $\Gamma$, $s, \mathcal{A}, \Sigma, H, E, I, J$ and $B$ defined in Eqs. (3), (41), (6), (8), (9), (10) and (20). These Riemann invariants can be computed when they are involved in the equations of a specific step (tags with outgoing arrows from the top and the left of the diagram). The explicit conditions labelling the different families of metrics in each step are presented inside diamonds. The labeled families of metrics are reported inside rectangles. 


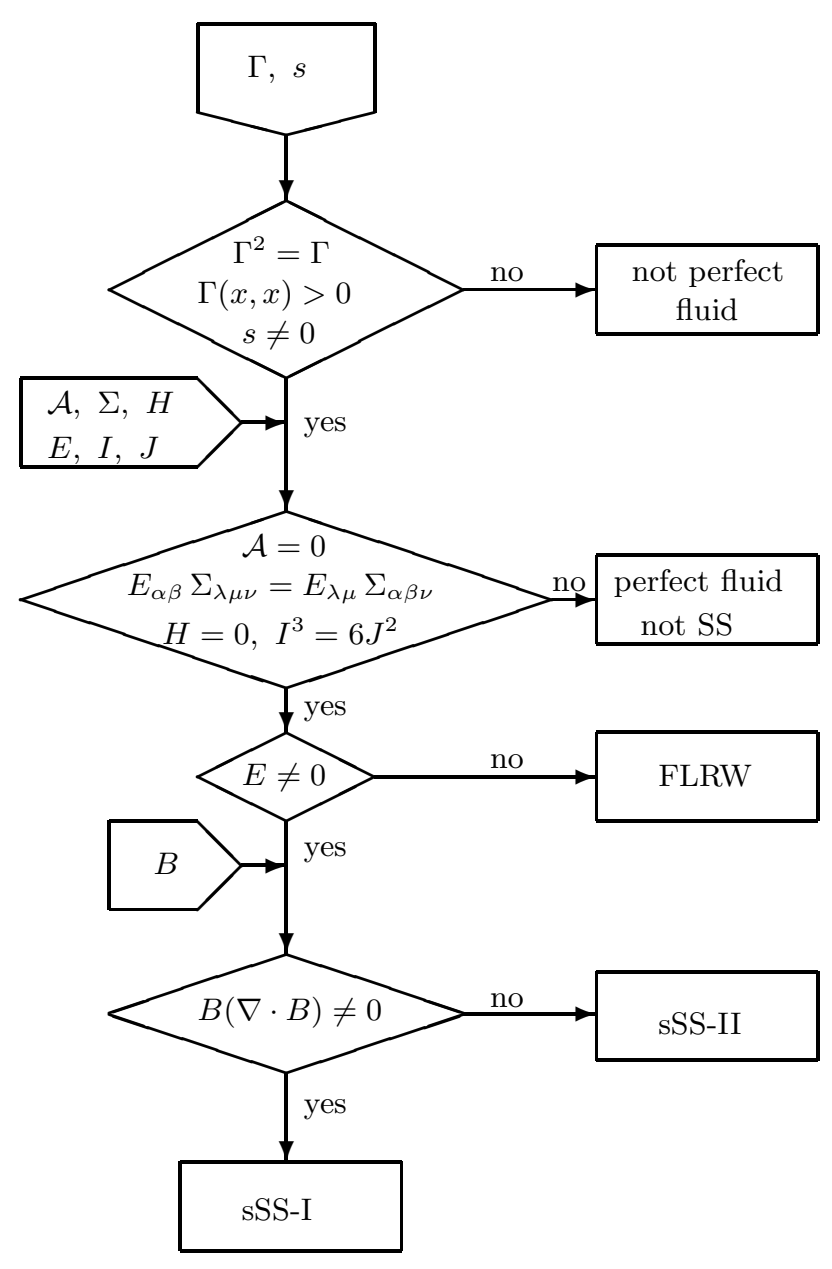

As commented in the Introduction, our IDEAL approach is an alternative to the Cartan-Brans-Karlhede method to analyze the equivalence of two metric tensors. This method is based on working in an orthonormal (or a null) frame fixed by the underlying geometry of the Riemann tensor. Nevertheless, the historic theorems that characterize locally flat Riemann spaces, Riemann spaces with a maximal group of isometries, and locally conformally flat Riemann spaces show that the determination of a Riemannian canonical frame is not necessary in labeling specific families of spacetimes. The conditions applied in these theorems involve explicit concomitants of the curvature tensor (Riemann, Weyl and Cotton tensors) and, consequently, they are IDEAL characterizations. We find a similar situation in characterizing other physically relevant families of spacetimes, such as the Stephani and the FLRW universes.

A suitable procedure is to analyze every particular case in order to understand the minimal set of elements of the curvature tensor that are necessary to label these geometries, an approach adapted to each particular geometry we want to characterize. This is the method we have achieved here in labeling the Szekeres-Szafron metrics, and it is also the one used in previous articles when characterizing different families of solutions 18 34].

\section{ACKNOWLEDGMENTS}

This work has been supported by the Spanish "Ministerio de Economía y Competitividad", MINECOFEDER project FIS2015-64552-P.

\section{Appendix A: Szekeres-Szafron metrics: coordinate functions and invariant scalars.}

The specific form of the coordinate functions $\alpha(t, z, x, y)$ and $\beta(t, z, x, y)$, and the expression of the pressure and energy density for Szekeres-Szafron metrics of classes I and II can be found in several papers [1] 2] [4]. Now we give these expressions by using the notation in Ref. 2] with a few changes. We also offer expressions for the simple Weyl eigenvalue and the expansion of the fluid.

\section{Szekeres-Szafron metrics of class I $(\beta, z \neq 0)$}

Coordinate functions:

$$
\begin{gathered}
e^{\alpha}=\phi, z+\phi \nu, z, \quad e^{\beta}=\phi S^{-1}, \quad \phi=\phi(t, z) \\
\mathrm{S}(z, x, y) \equiv \frac{1}{2} U(z)\left(x^{2}+y^{2}\right)+V_{1}(z) x+V_{2}(z) y+2 W(z) \\
\text { Pressure and energy density: } \\
p=-\left[\frac{2 \ddot{\phi}}{\phi}+\frac{\dot{\phi}^{2}}{\phi^{2}}+\frac{k(z)}{\phi^{2}}\right], \quad k(z) \equiv 4 U W-V_{1}^{2}-V_{2}^{2}-1
\end{gathered}
$$

$$
\rho=\frac{k^{\prime}+\left(\dot{\phi}^{2}+k\right)\left[(\ln \phi)_{z}+3 \nu, z\right]+2 \dot{\phi} \dot{\phi}, z}{\phi(\phi, z+\phi \nu, z)} .
$$

Simple Weyl eigenvalue:

$$
\omega=\frac{\phi \ddot{\phi}_{z}-\ddot{\phi} \phi,_{z}}{3 \phi\left(\phi,,_{z}+\phi \nu, z\right.} .
$$

Expansion:

$$
\theta=\frac{\dot{\phi}, z+\dot{\phi} \nu, z}{\phi, z+\phi \nu, z}+\frac{2 \dot{\phi}}{\phi}
$$

Szekeres-Szafron metrics of class II $(\beta, z=0)$

Coordinate functions:

$$
\begin{array}{r}
e^{\alpha}=\lambda+\phi P, \quad e^{\beta}=\phi C, \quad P=\mathrm{S} C, \\
\phi=\phi(t), \quad \lambda=\lambda(t, z), \quad \mathrm{S}=\mathrm{S}(z, x, y), \\
C(x, y) \equiv\left[1+\frac{k}{4}\left(x^{2}+y^{2}\right)\right]^{-1}, \quad k \equiv 0,1,-1, \\
\phi \ddot{\lambda}+\dot{\lambda} \dot{\phi}-\lambda \frac{\phi \ddot{\phi}+\dot{\phi}^{2}+k}{\phi}=U+k W .
\end{array}
$$


Pressure and energy density:

$$
\begin{array}{r}
p=-\left[\frac{2 \ddot{\phi}}{\phi}+\frac{\dot{\phi}^{2}}{\phi^{2}}+\frac{k}{\phi^{2}}\right], \\
\rho=\frac{2(\ddot{\phi} \lambda-\phi \ddot{\lambda})}{\phi(\lambda+\phi P)}+\frac{3 \dot{\phi}^{2}}{\phi^{2}}+\frac{3 k}{\phi^{2}} .
\end{array}
$$

Simple Weyl eigenvalue:

$$
\omega=\frac{\phi \ddot{\lambda}-\ddot{\phi} \lambda}{3 \phi(\lambda+\phi P)} .
$$

Expansion:

$$
\theta=\frac{\dot{\lambda}+\dot{\phi} P}{\lambda+\phi P}+\frac{2 \dot{\phi}}{\phi}
$$

\section{Appendix B: Some lemmas}

Lemma 10 For a strict Szekeres-Szafron metric of class $I$, the condition $\omega,_{z}=0$ implies $\nu, z x=\nu,_{z y}=0$.

Proof: Let us suppose that $\nu_{z x} \neq 0$. From the expression (A5) for $\omega$ we have that

$\omega,_{x}=\frac{-\omega \phi \nu,,_{z x}}{\phi,_{z}+\phi \nu, z}, \omega,_{z x}=\frac{-\omega,_{z} \phi \nu,{ }_{z x}}{\phi, z+\phi \nu, z}-\omega\left[\frac{\phi,_{, z x}}{\phi, z+\phi,_{, z}}\right]_{, z}$.

Furthermore, a straightforward calculation gives

$$
\left[\frac{\phi \nu, z x}{\phi, z+\phi \nu, z}\right]_{, x}=\frac{\phi \nu, z x}{\phi,_{z}+\phi,_{, z}}\left[\frac{\nu, r x x}{\nu_{, r x}}-\frac{\phi \nu, z x}{\phi,_{z}+\phi \nu, z}\right] .
$$

If $\omega_{, z}=0$, the expression (B1) implies that $\left[\frac{\phi \nu, z x}{\phi, z+\phi \nu, z}\right]_{, z}=0$. Then, by taking the derivative with respect to $z$ in Eq. (B2) we get that $\left[\ln \nu_{, z x}\right]_{, x z}=0$. If we compute this equation by taking into account the expression of $\nu$ in Eq. (A2), and equate the coefficients in the powers of $x$ and $y$, we get that $\nu_{, z x}=0$. Similarly we have that $\nu_{, z y}=0$.

Lemma 11 For a strict Szekeres-Szafron metric of class II, the condition $\omega,,_{z}=0$ implies,

$$
\begin{array}{r}
e^{\alpha}=\lambda(t)+\phi(t) \mathrm{S}(x, y), \\
\mathrm{S}(x, y) \equiv \frac{1}{2}\left(x^{2}+y^{2}\right)+c_{1} x+c_{2} y+2 c,
\end{array}
$$

where $c_{i}$ and $c$ are constants. Consequently $\xi=\partial_{z}$ is a Killing vector.

Proof: Without loss of generality we can take $W(z)=0$ in the expression (A2) of the function $S$ of a metric of class II [1]. From the expression (A13) of $\omega$, and taking into account that $\phi \equiv \phi(t)$, it holds that $\omega,_{z}=0$ is equivalent to $(\phi \omega)_{, z}=0$. A straightforward calculation shows that this condition can be stated as

$G,,_{z}\left[\lambda C^{-1}+\phi S\right]=G\left[\lambda,,_{z} C^{-1}+\phi S, z\right], \quad G \equiv \phi \ddot{\lambda}-\ddot{\phi} \lambda$. If we expand the expression above and equate the coefficients in the different powers of $x$ and $y$, we have that

$$
\frac{G_{, z}}{G}=\frac{\lambda_{, z}}{\lambda}=\frac{U^{\prime}}{U}=\frac{V_{1}^{\prime}}{V_{1}}=\frac{V_{2}^{\prime}}{V_{2}} .
$$

Integrating these equations we get $(\lambda / U)_{, z}=(P / U)_{, z}=$ 0 . Thus, a redefinition of the coordinate $z$ leads to the lemma.

Lemma 12 For a Szekeres-Szafron metric of class I, the condition $\rho_{z}=0$ leads necessarily to a FLRW universe.

Proof: If $\rho, z=0$, then we have $\dot{\rho}_{, z}=0$ and, from the conservation condition, we get that $\theta_{, z}=0$. On the other hand, from Eq. (25), we get that $\rho_{, z}=0$ is equivalent to $\omega_{, z}+[\ddot{\phi} / \phi]_{, z}=0$. If we expand this last condition, and use $\dot{\theta}_{, z}=0$ to replace $\ddot{\phi}, z z$, we obtain that $\rho_{, z}=0$ implies $\phi \dot{\phi}_{, z}-\dot{\phi} \phi_{, z}$. Then, from Eq. (A5), $\omega=0$ and the solution is a FLRW universe.

Lemma 13 For a strict Szekeres-Szafron metric of type $I$, condition $\dot{Q}=0$ implies $\nu_{z x}=\nu, z y=0$, where

$$
\begin{aligned}
Q & =-\nu, z\left[\frac{F,_{z z}}{F, z}+6 \frac{\phi, z}{\phi}\right]-3 \nu,,_{z} \nu, z+ \\
& +\left[\frac{\phi, z z}{\phi}-\frac{\phi, z}{\phi} \frac{F, z z}{F,_{z}}-4 \frac{\phi, z \phi, z}{\phi^{2}}\right], \quad F \equiv \frac{\ddot{\phi}}{\phi} .
\end{aligned}
$$

Proof: The condition $\dot{Q}=0$, implies $h(\mathrm{~d} \dot{Q})$. If we expand this last expression we get:

$$
\left[\frac{F_{, z z}}{F_{, z}}+6 \frac{\phi_{, z}}{\phi}\right]_{, t} h\left(\mathrm{~d} \nu_{, z}\right)=0 .
$$

Thus, either $h\left(\mathrm{~d} \nu_{, z}\right)=0$ or $\left[\frac{F_{, z z}}{F, z}+6 \frac{\phi_{, z}}{\phi}\right]_{, t}=0$. Then, if $h\left(\mathrm{~d} \nu_{, z}\right) \neq 0$, the condition $\dot{Q}=0$ is equivalent to the two equations

$\left[\frac{F_{, z z}}{F_{, z}}+6 \frac{\phi_{, z}}{\phi}\right]_{, t}=0, \quad\left[\frac{\phi_{, z z}}{\phi}-\frac{\phi_{, z}}{\phi} \frac{F_{, z z}}{F_{, z}}-4 \frac{\phi_{, z} \phi_{, z}}{\phi^{2}}\right]_{, t}=0$.

The first equation can be partially integrated and we have that two functions $a(z)$ and $b(t)$ exist such that

$$
F_{, z} \phi^{6}=e^{a(z)} e^{b(t)} .
$$

Putting this into the second equation, we have that the function $\phi$ necessarily factorizes, and we obtain a FLRW universe. 
[1] A. Krasiński, Inhomogeneous Cosmological Models (Cambridge University Press, Cambridge, England, 1997).

[2] A. Krasiński and J. Plebański, An Introduction to General Relativity and Cosmology (Cambridge University Press, Cambridge, England, 2012).

[3] G. Ellis, R. Maartens, and M. A. C. MacCallum, Relativistic Cosmology (Cambridge University Press, Cambridge, England, 2012).

[4] D. A. Szafron, J. Math. Phys. 18, 1673 (1977).

[5] P. Szekeres, Commun. Math. Phys. 41, 55 (1975).

[6] W. B. Bonnor, Commun. Math. Phys. 51, 191 (1976).

[7] W. B. Bonnor, A. H. Sulaiman, and N. Tomimura, Gen. Relativ. Gravit. 8, 549 (1977).

[8] B. K. Berger, D. M. Eardley, and D. W. Olson, Phys. Rev. D 16, 3086 (1977).

[9] S. W. Goode and J. Wainwright, Phys. Rev. D 26, 3315 (1982).

[10] C. Hellaby, Classical Quantum Gravity 34, 145006 (2017).

[11] I. Georg and C. Hellaby, Phys. Rev. D 95, 124016 (2017).

[12] J. Wainwright, J. Math. Phys. 18, 672 (1977).

[13] D. A. Szafron and C. Collins., J. Math. Phys. 20, 2354 (1979).

[14] C. Collins and D. A. Szafron, J. Math. Phys. 20, 2347 (1979).

[15] A. Barnes and R. R. Rowlingson, Classical Quantum Gravity 6, 949 (1989).

[16] H. Takeno, Prog. Theor. Phys. 8, 317 (1952).

[17] H. Takeno, The Theory of Spherically Symmetric SpaceTimes, Revised ed. (Hiroshima University, Hisoshima, 1966).

[18] J. J. Ferrando and J. A. Sáez, Classical Quantum Gravity 27, 205024 (2010).

[19] J. J. Ferrando and J. A. Sáez, Classical Quantum Gravity 34, 045002 (2017).

[20] J. J. Ferrando and J. A. Sáez, Classical Quantum Gravity 14, 129 (1997).

[21] J. J. Ferrando and J. A. Sáez, Classical Quantum Gravity 15, 1323 (1998).

[22] J. J. Ferrando, J. A. Morales, and J. A. Sáez, Classical Quantum Gravity 18, 4939 (2001).

[23] J. J. Ferrando and J. A. Sáez, Classical Quantum Gravity 19, 2437 (2002).

[24] J. J. Ferrando and J. A. Sáez, Gen. Relativ.Gravit. 35, 1191 (2003).

[25] J. J. Ferrando and J. A. Sáez, Classical Quantum Gravity 20, 5291 (2003).

[26] J. J. Ferrando and J. A. Sáez, J. Math. Phys. 45, 652 (2004).
[27] J. J. Ferrando and J. A. Sáez., Gen. Relativ.Gravit. 36, 2497 (2004).

[28] B. Coll, J. J. Ferrando, and J. A. Sáez, J. Math. Phys. 47, 062503 (2006).

[29] J. J. Ferrando and J. A. Sáez, J. Math. Phys. 47, 112501 (2006).

[30] J. J. Ferrando and J. A. Sáez., Gen. Relativ. Gravit. 39, 2039 (2007).

[31] J. J. Ferrando and J. A. Sáez, J. Math. Phys. 48, 102504 (2007).

[32] J. J. Ferrando and J. A. Sáez, Classical Quantum Gravity 26, 075013 (2009).

[33] J. J. Ferrando and J. A. Sáez, Classical Quantum Gravity 27, 205023 (2010).

[34] J. J. Ferrando and J. A. Sáez, Gen. Relativ. Gravit. 46, 1703 (2014).

[35] G. Canepa, C. Dappiaggi, and I. Khavkine, Classical Quantum Gravity 35, 035013 (2018).

[36] E. Cartan, Leçons sur le Géométrie des Espaces de Riemann (Gauthier-Vilar, Paris, 1946).

[37] C. H. Brans, J. Math. Phys. 6, 94 (1965).

[38] A. Karlhede, Gen. Relativ. Gravit. 12, 693 (1980).

[39] A. García-Parrado-Gómez-Lobo and J. A. ValienteKroon, Phys. Rev. D 75, 024027 (2007).

[40] A. García-Parrado-Gómez-Lobo, Phys. Rev. D 92, 124053 (2015).

[41] A. García-Parrado-Gómez-Lobo, Classical Quantum Gravity 33, 175005 (2016).

[42] B. Coll, in Relativistic Geodesy: Foundations and Application. Proceedings of 609 WE-Heraeus Seminar (2016) (to be published) arXiv:1712.05712.

[43] G. Y. Rainich, Trans. Math. Soc. 27, 106 (1925).

[44] B. Coll and J. J. Ferrando, J. Math. Phys. 30, 2918 (1989).

[45] E. Stephani, H. Kramer, M. A. H. McCallum, C. Hoenselaers, and E. Hertl, Exact Solutions of Einstein's Field Equations (Cambridge University Press, Cambridge, England, 2003).

[46] C. Bona, B. Coll, and J. A. Morales, J. Math. Phys. 33, 670 (1992).

[47] J. Wainwright, Gen. Relativ. Gravit. 8, 797 (1977).

[48] J. Plebański, Acta Phys. Polon. 26, 963 (1964).

[49] B. Coll, J. J. Ferrando, and J. A. Sáez, Gen. Relativ. Gravit. 49, 66 (2017).

[50] A. Krasiński, H. Quevedo, and R. A. Sussman, J. Math. Phys. 38, 2602 (1997).

[51] B. Coll and J. J. Ferrando, Some Topics on General Relativity and Gravitational Radiation: Proceedings of Spanish Relativity Meeting-96, Valencia (Ed. Frontières, París, 1997). 